\title{
The Impact of a Mediterranean Diet on the Gut Microbiome in Healthy Human Subjects: A Pilot Study
}

\author{
Jared J. Rejeski ${ }^{a}$ Farra M. Wilson $^{a}$ Ravinder Nagpal ${ }^{b}$ Hariom Yadav ${ }^{c, d}$ \\ Richard B. Weinberg ${ }^{\text {a, }}$ e \\ aDepartment of Internal Medicine-Gastroenterology, Wake Forest School of Medicine, Winston-Salem, NC, USA; \\ ${ }^{b}$ Department of Nutrition and Integrative Physiology, Florida State University, Tallahassee, FL, USA; 'Department \\ of Nutrition and Integrative Physiology, Wake Forest School of Medicine, Winston-Salem, NC, USA; dDepartment \\ of Microbiology and Immunology, Wake Forest School of Medicine, Winston-Salem, NC, USA; 'Department of \\ Physiology \& Pharmacology, Wake Forest School of Medicine, Winston-Salem, NC, USA
}

\section{Keywords}

Microbiome $\cdot$ Microflora $\cdot$ Nutrition

\begin{abstract}
Background and Aims: Despite the reported salutary benefits of a Mediterranean diet (MD) on a wide variety of health conditions, the specific microbial changes associated with an MD within the gastrointestinal (Gl) tract are not well studied. Specifically, although population and survey-based studies have shown microbial changes, there are no published data on how an MD alters the gut flora in a controlled setting. Methods: We recruited 10 healthy subjects, each of whom gave a stool sample at baseline and then was provided with prepared meals of a "typical" American diet; after 2 weeks, a second stool sample was collected. All subjects were then provided with prepared meals based on the MD for another 2 weeks, followed by a final stool sample collection. Stool samples were batch analyzed with DNA extraction, and sequencing libraries were generated. Measures of bacterial diversity, species richness, and enterotypes were performed. Results: All ten subjects tolerated the diets well. Bacterial diversity increased with an MD, as measured by alpha diversity via the Simpson index. Furthermore, there
\end{abstract}

karger@karger.com www.karger.com/dig

Karger $\stackrel{\text { ' }}{5}$

GOPEN ACCESS
(C) 2021 The Author(s)

Published by S. Karger AG, Basel

This is an Open Access article licensed under the Creative Commons Attribution-NonCommercial-4.0 International License (CC BY-NC) (http://www.karger.com/Services/OpenAccessLicense), applicable to the online version of the article only. Usage and distribution for commercial purposes requires written permission. were significant differences in 5 bacterial genera between the 2 diets. Conclusion: This small pilot study of controlled diets demonstrates that the MD can rapidly alter the gut microbiome in healthy subjects at the level of global microbial diversity and individual genera. These data confirm the findings of previous observational studies and establish the feasibility of conducting longer term studies on the impact of the MD on the flora of the Gl tract and its relationship to digestive diseases.

(C) 2021 The Author(s) Published by S. Karger AG, Basel

\section{Introduction}

The microbial population of the gastrointestinal (GI) tract is enormous, and our understanding of how it impacts the human body and disease is rapidly evolving. Trends predictive of health and disease have been identified in both specific communities of bacteria and the diversity of these communities [1]. Logically, habitual diet plays a major role in the microbial composition of the GI tract, and thus, the impact of diet on the microbiota has

Registry website: https://clinicaltrials.gov/ct2/show/NCT03269032.
Correspondence to:

Jared J. Rejeski, jarejesk@wakehealth.edu 
been an attractive target for clinical research $[2,3]$. The Mediterranean diet (MD) is an ancient dietary pattern that is consumed by a significant proportion of the world's population. The MD not only is known to have beneficial health effects - including reduction in risk for cardiovascular disease, type 2 diabetes, fatty liver disease, certain cancers, and neurodegenerative disorders [4] - but recently has been shown to impact microbial diversity and microbiome composition in observational studies in humans and diet modification studies in nonhuman primates [5-10].

The microbiome and its dysregulation have also been implicated in the etiology of digestive diseases, specifically the pathogenesis of irritable bowel syndrome (IBS), the most prevalent GI disorder [11-13]. Treatment of IBS poses many diagnostic and therapeutic challenges; however, recent evidence links adherence to an MD and improvement in IBS-type symptoms [14]. Therefore, delineating the changes in the microbiome caused by an MD is warranted. While prior studies have quantified changes in the microbiome as they relate to patient-reported diets, no human studies have specifically demonstrated how a controlled delivery of an MD can affect an individual's microbiome composition or diversity. Therefore, as a pilot study for future research on how the MD affects IBS, we performed a small dietary modification study to examine how a standardized MD changes the microbiota when fed after a baseline standardized "American" diet (AD). We hypothesized that the MD would produce more varied microbiota composed of favorable enterotypes than in an $\mathrm{AD}$.

\section{Methods}

\section{Study Details and Participants}

We recruited 10 healthy individuals who were $\geq 21$ years old and were not following any prescribed dietary regimens via posted notices. Respondents who reported that they were clinically healthy, had no active medical problems, and were not taking any prescribed or over-the-counter medications were scheduled for a screening visit at our medical center's Clinical Research Unit (CRU).

\section{Study Design}

At the screening visit, informed consent was obtained from each subject by a member of the study team. Subjects then had a nutritional consultation with the CRU research dietitian to review their dietary preferences, record any food allergies or intolerances, and review study procedures. Basal caloric expenditure was measured by indirect calorimetry using a metabolic cart in 8 of the subjects; in 2 subjects, basal caloric expenditure was estimated by
Table 1. Nutrient composition of each of the 2 week diets

\begin{tabular}{lll}
\hline Nutrient (\% of total energy) & AD, \% & MD, \% \\
\hline Carbohydrate/alcohol $^{*}$ & 50 & 51 \\
Protein & 15 & 17 \\
Total fat & 35 & 32 \\
$\quad$ Saturated & 11.2 & 6.7 \\
$\quad$ Monounsaturated & 12.1 & 18.1 \\
$\quad$ Polyunsaturated & 8.2 & 4.7 \\
\hline
\end{tabular}

* Alcohol (red wine, 5 ounces/day) included in MD only. MD, Mediterranean diet; AD, American diet.

the Harris-Benedict equation. A stool sample was collected or arranged during this visit. On a mutually agreeable date, subjects began an individually tailored isocaloric $\mathrm{AD}$ prepared in the CRU metabolic kitchen and provided as prepackaged trays and containers in soft coolers. Subjects returned to the CRU to collect food 3 times weekly and to assess adherence to the provided meals by CRU staff. Two weeks after starting the AD, the subjects returned to the CRU with a stool sample and then began the Mediterraneanstyle diet, provided as described for the AD, for the next 2 weeks. On day 28 of the study, subjects returned to the CRU to deliver a final stool sample. The nutritional composition of the diets and sample menus are given in Tables 1 and 2, respectively.

\section{Gut Microbiota Measurement}

Gut microbiome was examined as per our previously described methods $[9,15,16]$. In brief, the bacterial microbiome composition was analyzed in accordance with the Earth Microbiome Project benchmarked protocol (http://www.earthmicrobiome.org), by adopting a bar-coded high-throughput sequencing approach as described by Caporaso et al. [17]. The fecal samples were stored at $-70^{\circ} \mathrm{C}$ as soon as they were delivered to the CRU. The DNA was extracted using the MoBio PowerFecal Kit (Qiagen, CA, USA) according to the manufacturer's instructions. The V4 hypervariable region of the $16 \mathrm{~S}$ rDNA gene was PCR amplified using the universal primer pair 515F (bar-coded) and 806R. Both the forward and reverse primer sequences also contained appropriate Illumina adapters, primer pad, and 2-bp linker sequences, with forward primer containing a 12-bp error-correcting barcode unique to each sample to achieve multiplex capability. All primers were synthesized at the Integrated DNA Technologies (IDT Inc., San Jose, CA, USA). The PCR was performed, as described previously $[9,15$, 18]. The resulting amplicons were cleaned up by using Agencourt $^{\circledR}$ AMPure ${ }^{\circledR}$ XP magnetic purification beads (Beckman Coulter, Brea, CA, USA) and quantified by using a Qubit-3 Fluorometer (Invitrogen, Carlsbad, CA, USA) and dsDNA HS assay kit (Life Technologies, Carlsbad, CA, USA); the final amplicon library was made according to methods of Caporaso et al. [17]. The purified PCR products were pooled in equimolar concentrations and sequenced on one $2 \times 300$-bp Illumina MiSeq run (Illumina Inc., San Diego, CA, USA) for paired-end sequencing. The sequencing quality control was executed with onboard MiSeq Control Software and MiSeq Reporter (Illumina Inc.), and the obtained sequences were de-multiplexed, quality-filtered, clustered, and taxonomically annotated against Greengenes reference database with 
RDP Classifier using the Quantitative Insights into Microbial Ecology (http://qiime.org/) software package, as described previously $[9,15,18,19]$. A $97 \%$ sequence similarity cutoff was used to cluster operational taxonomic units (OTUs). Negative controls were mock specimens that contained all reagents, including barcoded primers but without any extracted DNA that went through PCR and sequencing procedures. To avoid the effect of DNA extraction or PCR conditions and primers on microbial community composition recovered by amplicon sequencing, all samples were batch-processed and analyzed to minimize such bias if any. A total of $1,365,088$ sequences (minimum 21,793 ; maximum 72,742 ; median 43,184; standard deviation 12,957; reads per sample) were obtained after quality-filtering using default parameters. To avoid the bias of sequencing depths, the obtained sequences were rarefied to the lowest number (21,793 reads) of sequences for downstream analyses. To avoid the bias of sequencing errors or lowlevel contaminations, the OTUs with count $<4$ and prevalence less than $10 \%$ were removed from subsequent analyses. Of 4,795 total OTUs originally obtained, 3,457 OTUs were retained after these steps. The resultant data of taxon proportions were subjected to the total sum scaling, and the taxa with $<1 \%$ mean relative abundance were further excluded from subsequent comparative analyses.

\section{Data Analysis}

The beta diversity of the microbiome was assessed using the Bray-Curtis dissimilarity index and was visualized with the principal coordinate analysis in $\mathrm{R}$ statistical software package (version 3.6.1; www.r-project.org). Difference in beta diversity was calculated by permutational multivariate analysis of variance, a permutation-based multivariate analysis of variance to a matrix of pairwise distance to assess the intergroup and intragroup distance. Alpha diversity indices were calculated within Quantitative Insights into Microbial Ecology. The random forest supervised learning model was applied on normalized data within R (model_randomForest; $70 \%$ training and $30 \%$ testing modules; trees $=500$ ) to identify the major bacterial taxa whose abundance is affected by the intervention (OOB error: 0.5). Alpha diversity indices and the bacterial proportions were compared using the Kruskal-Wallis test followed by the Mann-Whitney multiple pairwise comparison test. Discriminative features, that is, the bacterial taxa, uniquely modulated by the dietary intervention and driving differences between different groups were identified by using the biomarker discovery algorithm (linear discriminatory analysis [LDA] effect size [LEfSe]) with an LDA score $>3.0$ and $p$ value $<0.05$ [20]. Significance threshold was set at $p<0.05$, unless otherwise specified.

\section{Results}

Demographic, anthropometric, and basal metabolic rate data for the 10 subjects are provided in Table 3. Six subjects were male, and 4 subjects were female; 7 subjects were Caucasian, and 3 subjects were Asian. Overall, there were no significant differences between ethnicity and gender. The average age of the subjects was $31.8 \pm 3.3$ years. The average BMI was $22.9 \pm 0.9$ at baseline and 22.8

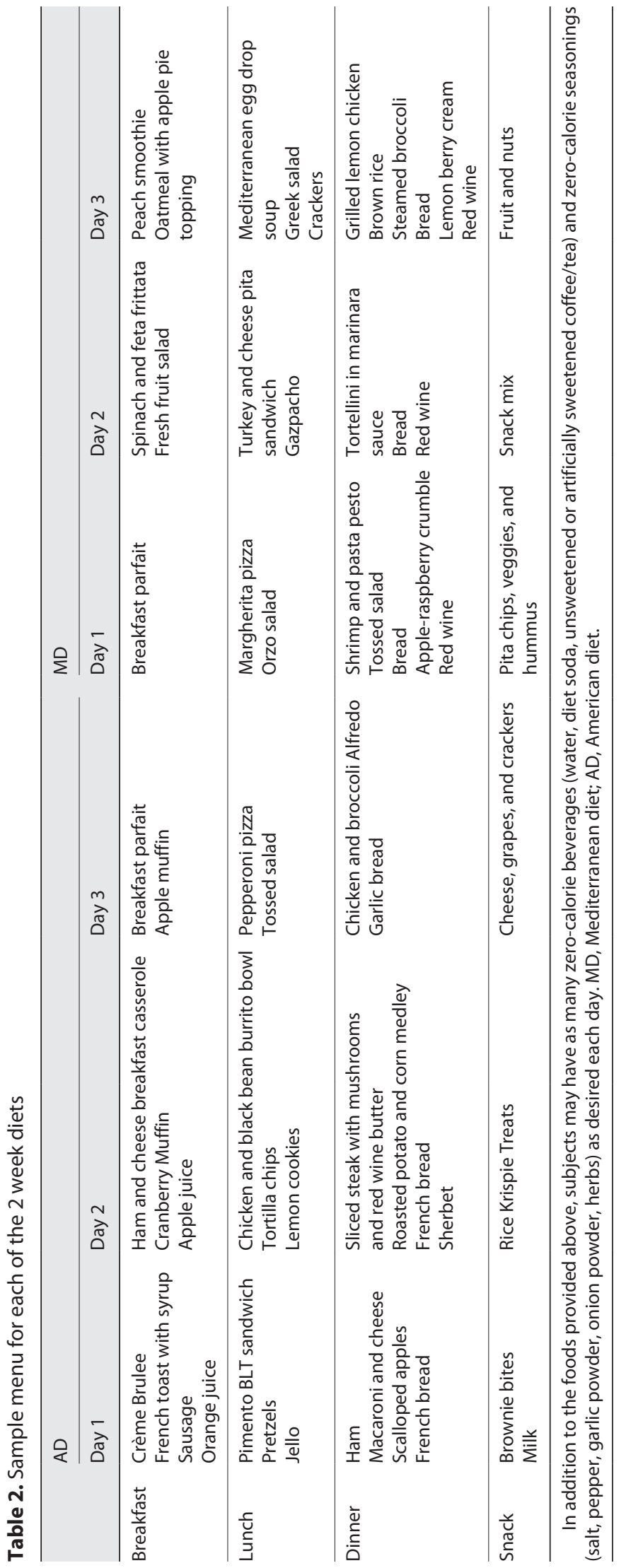

Digestion 2022;103:133-140

DOI: $10.1159 / 000519445$ 
Table 3. Demographic, anthropometric, and basal metabolic rate data for the 10 subjects

\begin{tabular}{lllllll}
\hline Subject & $\begin{array}{l}\text { Age, } \\
\text { years }\end{array}$ & Sex & Race & $\begin{array}{l}\text { BMI } \\
\text { Baseline }\end{array}$ & $\begin{array}{l}\text { BMI } \\
\text { Week } 4\end{array}$ & $\begin{array}{l}\text { Resting metabolic } \\
\text { rate (kcal) }\end{array}$ \\
\hline 1 & 60 & F & Caucasian & 22.2 & 21.6 & 1,104 \\
2 & 26 & M & Caucasian & 23.9 & 23.5 & $1,912^{*}$ \\
3 & 26 & M & Caucasian & 22.2 & 22.2 & 1,727 \\
4 & 28 & M & Caucasian & 21.6 & 20.9 & 1,768 \\
5 & 26 & M & Asian & 24.3 & 25.3 & $2,014^{*}$ \\
6 & 26 & M & Asian & 20.5 & 20.6 & 1,417 \\
7 & 33 & M & Caucasian & 24.4 & 23.5 & 1,694 \\
8 & 33 & F & Caucasian & 29.4 & 29.6 & 1,414 \\
9 & 25 & F & Asian & 19.9 & 20.3 & 1,177 \\
10 & 35 & F & Caucasian & 20.4 & 20.2 & 1,303 \\
\hline Mean \pm SE & $31.8 \pm 3.3$ & - & - & $22.9 \pm 0.9$ & $22.8 \pm 0.9$ & $1,553 \pm 99$ \\
\hline
\end{tabular}

BMI, body mass index. * Indicates RMR estimated from Harris-Benedict equation.

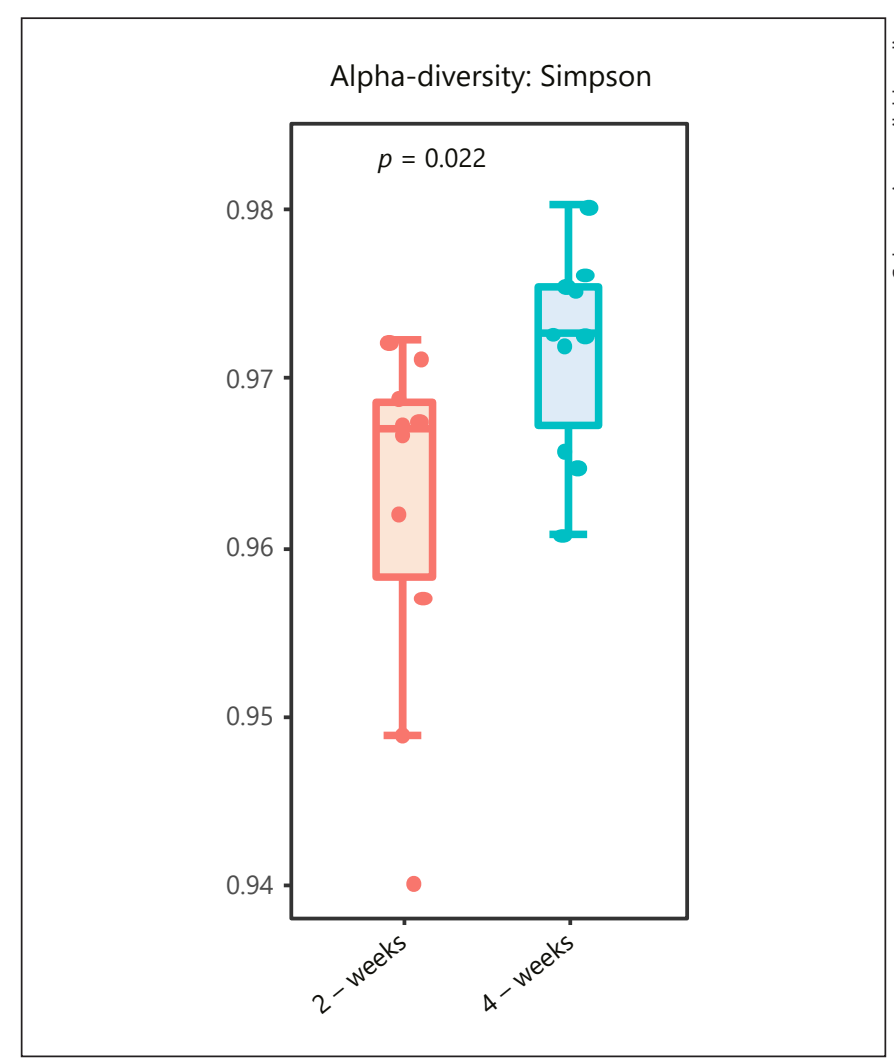

Fig. 1. Box plot showing the comparison of alpha diversity estimates by using a Simpson index at week 2 after the $\mathrm{AD}$ and at week 4 after the MD. MD, Mediterranean diet; AD, American diet.

\pm 0.9 at week 4 . There was no significant change in BMI between the baseline visit and study completion at week 4. Average resting energy expenditure as determined by calorimetry or calculation of the resting metabolic rate was $1,453 \pm 99 \mathrm{kcal}$. Subjects reported good adherence to the diets; no significant adverse events were reported.

Using a Simpson index to evaluate the alpha diversity between the completion of the 2 standardized dietary interventions (week 2 vs. week 4), there was a significant increase in microbial diversity (Fig. 1). Furthermore, 5 key genera were identified as showing statistically significant signal differences via a random forest with a mean decrease in accuracy analysis between the 2 standardized dietary interventions (Fig. 2). Specifically, Akkermansia, Lactococcus, and Lachnospira increased, whereas Coprococcus and SMB53 decreased. There were further signals within several other genera that did not meet statistical significance including a decrease in Anaerostipes, Collinsella, and Eubacterium as well as an increase in Phascolarctobacterium and Faecalibacterium. Evaluation with a Shannon index shows that between baseline and the post$\mathrm{AD}$ stool, there was a trend toward less diversity of the microbiome; there was subsequently a trend toward greater diversity than at both baseline and post-AD after the MD intervention (Fig. 3).

Via use of a LEfSe algorithm and LDA, classes that were statistically different with $\log >3$ between the samples at week 2 (after AD) and week 4 (after MD) were identified. The phylotypes which were identified as key determinants of the increased diversity, as measured by the Simpson index, seen after consumption of the MD were identified as Lachnospira, Sutterella, Burkholderiales, Alcaligenaceae, and Betaproteobacteria, whereas SMB53 was the sole statistically significant determinant of the signal seen after an AD. Figure 4 illustrates the LEfSe and LDA. Figures 5 and 6 show the changes seen at the level of major bacterial phyla and genera and bacte- 


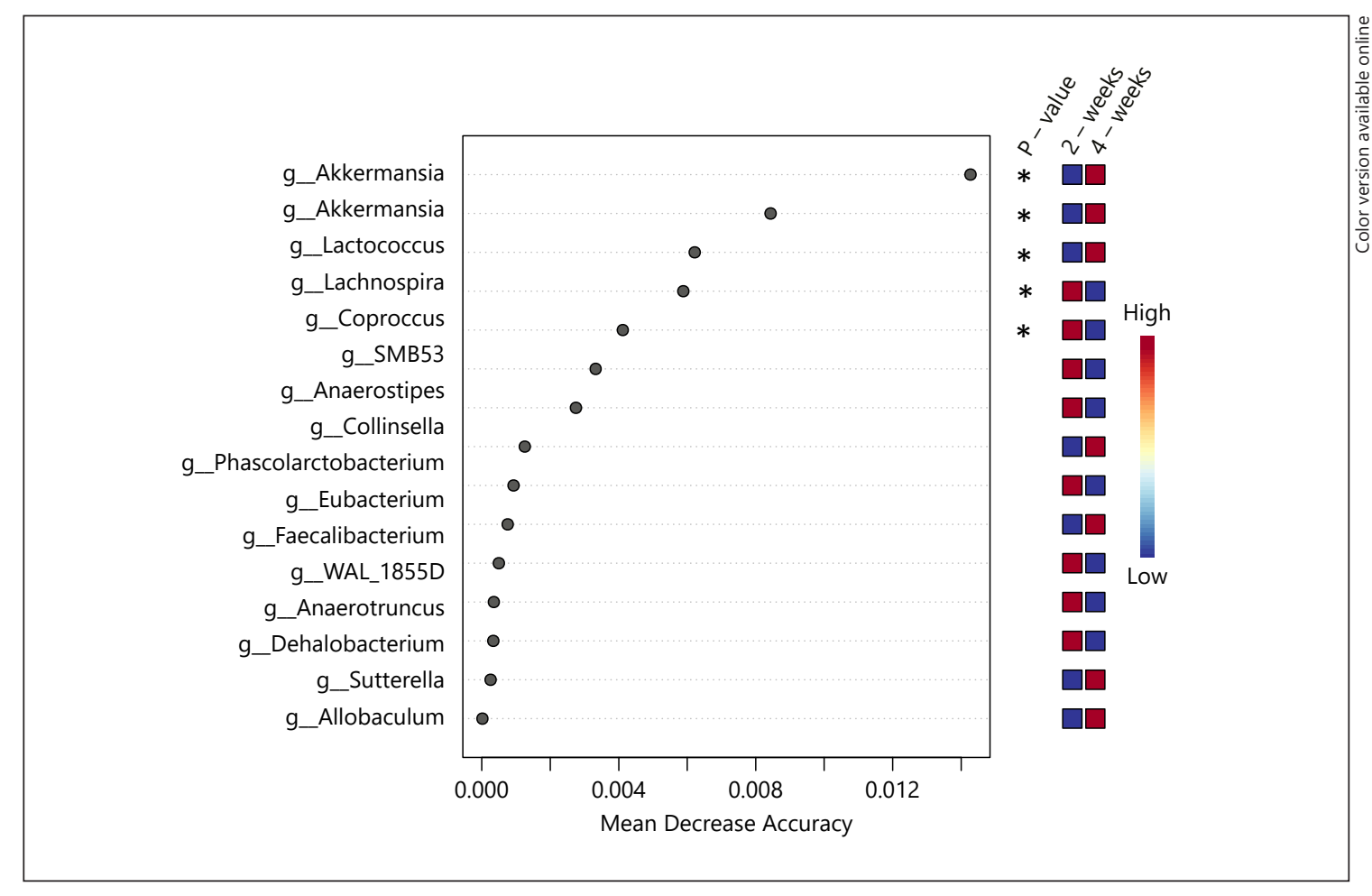

Fig. 2. Plot of mean decrease in accuracy using a random forest classification to identify signatures among key genera among the participants at week 2 after the $\mathrm{AD}$ and at week 4 after the MD; 5 of which reached statistical significance at $p<0.05$ (top 5). MD, Mediterranean diet; AD, American diet.

rial phenotypes, respectively. The trend seen within major phyla (Fig. 5) shows reduction in Firmicutes, increase in Bacteroidetes, and overall increased diversity on a phyla level, whereas differences at week 2 and week 4 between genera are more subtle with only slight increases in Bacteroides, Faecalibacterium, and Lachnospiraceae, while Blautia and Ruminococcus decreased (Fig. 5). The proportion of aerobic bacteria significantly increased from week 2 to week 4 , and overall phenotype showed a trend toward less Gram-positive phenotypes (Fig. 6).

\section{Discussion}

Dietary modification of the microbiome has the potential to treat disease. Dietary patterns that are rich in variety are associated with a more diverse microbiome, 1 of the key characteristics in a healthy microbiota. Evidence suggests diet as the most important factor in the composition of the microbiome, but no controlled studies have examined specifically how an MD affects the human microbiome [21]. To the best of our knowledge, this study is therefore the first in examining, via high-through-

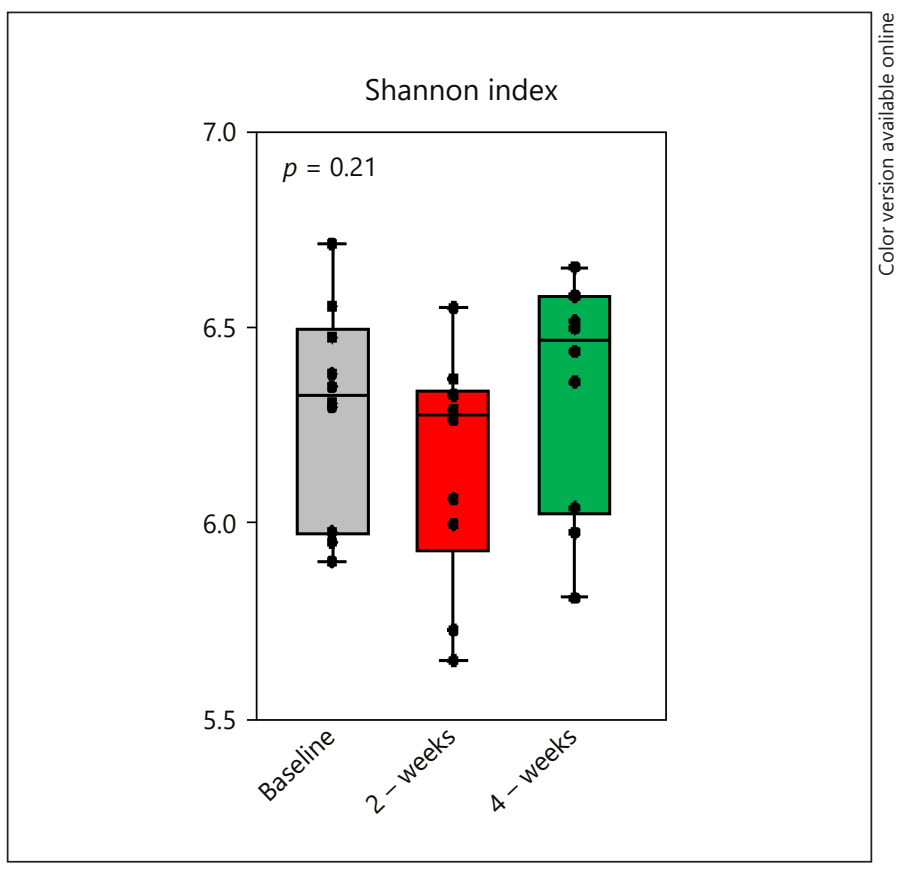

Fig. 3. Box plot showing the comparison of diversity estimates by using a Shannon index at baseline collection, week 2 after the AD and at week 4 after the MD. MD, Mediterranean diet; AD, American diet. 
Fig. 4. LEfSe showing microbial classification of stool samples at week 2 after the AD and at week 4 after the MD. The cladogram on the left shows a phylogenetic classification of differences by each successive circle at the phylum, class, family, and genus level between the week 2 and week 4 stool collections. Highlighted elements in red indicate prominence at week 2 collection and in green indicate prominence in week 4 collection. Major differences noted in top middle with marked differences within Sutterella (c), Alcaligenaceae (d), and Burkholderiales (e). Each circle size represents the relative abundance within the sample. The LDA score is shown on the histogram to the right. $\mathrm{MD}$, Mediterranean diet; $\mathrm{AD}$, American diet; LDA, linear discriminatory analysis; LEfSe, linear discriminatory analysis effect size.
Fig. 5. Bar graph showing the major abundance of bacterial phyla and genera as measured among the study participants at week 2 after the AD and at week 4 after the MD. $\mathrm{MD}$, Mediterranean diet; $\mathrm{AD}$, American diet.
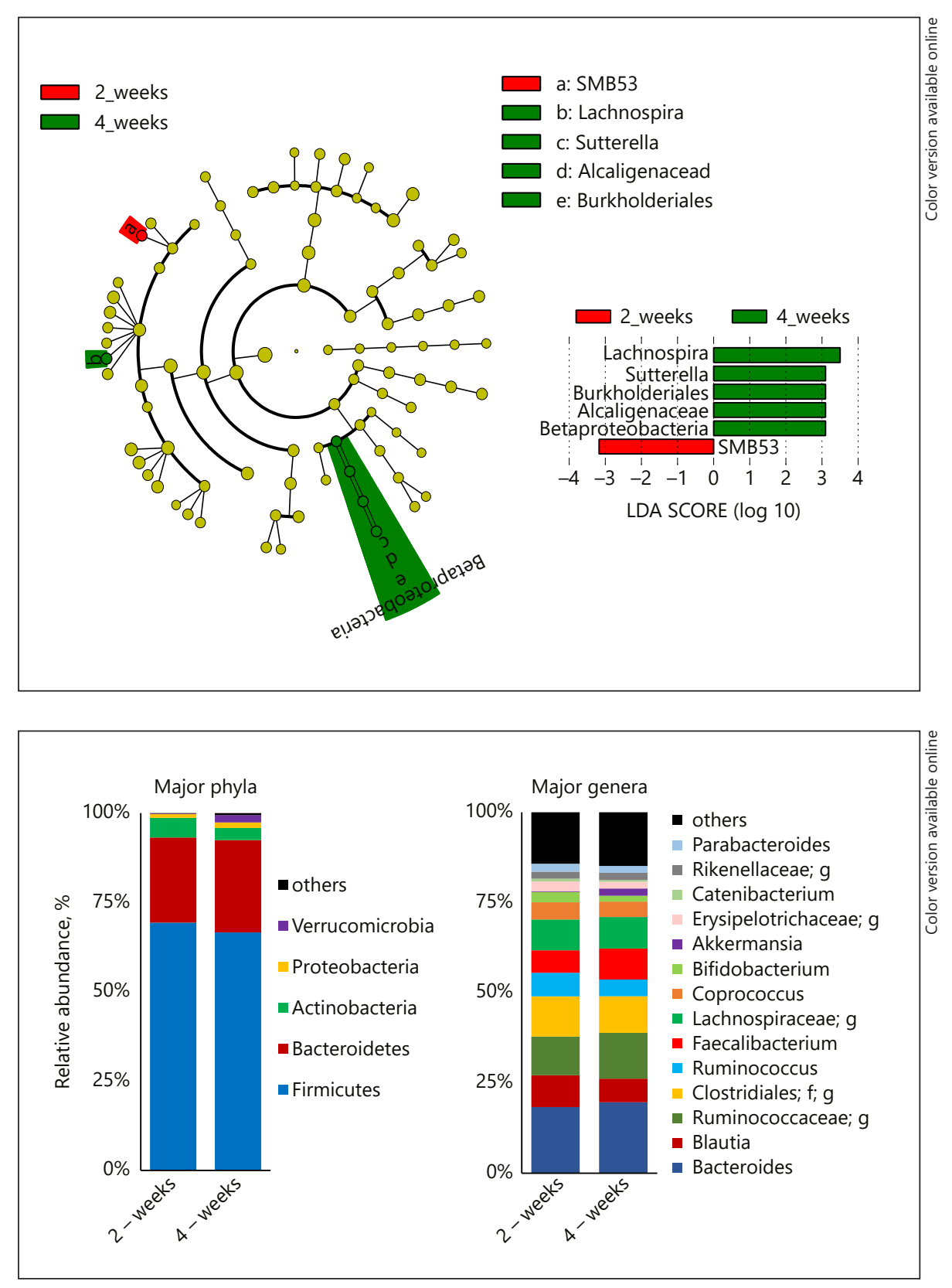

put DNA sequencing, the impact on the microbiome of an MD from both baseline and an $\mathrm{AD}$ with strictly controlled diets within a standardized setting among healthy adults.

Standardizing diets from an $\mathrm{AD}$ to an MD showed a significant rise in alpha diversity indicating a defined positive change in just 2 weeks (Fig. 1). This increased microbial diversity, or trend away from "dysbiosis," contrasts results of survey-based and population studies, including 1 of the largest efforts, the NU-AGE project $[5,8,22]$. However, a decrease in the ratio of Firmicutes/Bacteroide- tes, the most prevalent phyla in the human gut, was seen with a standardized MD (Fig. 5) which mirrors changes seen in survey-based studies [5]. The Firmicutes/Bacteroidetes ratio has been linked to obesity with lower levels of Bacteroidetes associated with obesity and higher levels seen after successful weight loss [23]. Interestingly, similar to our study among healthy adult subjects, in a longterm standardized dietary intervention of MD versus an $\mathrm{AD}$ among nonhuman primates, both increased microbial diversity (via Shannon diversity index) and decreased Firmicutes/Bacteroidetes ratio were seen [9]. 


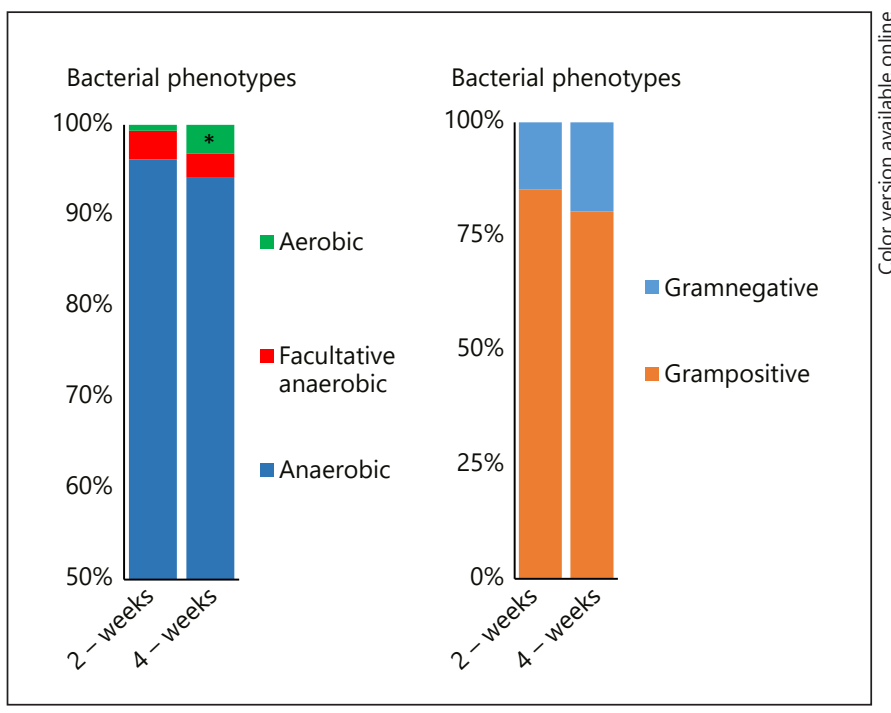

Fig. 6. Bar graph showing the major abundance of bacterial phenotypes as measured among the study participants at week 2 after the $\mathrm{AD}$ and at week 4 after the MD. MD, Mediterranean diet; $\mathrm{AD}$, American diet.

While not reaching overall statistical significance, among these healthy individuals, a standardized $\mathrm{AD}$ showed a trend of decreasing diversity, while an MD increased diversity over both baseline and an $\mathrm{AD}$ via use of a Shannon index (Fig. 3). Despite this, statistically significant changes occurred among several identified genera. The marked increase in Akkermansia after MD follows recent evidence suggesting the benefit of these genera with respect to metabolism and glucose homeostasis, among other effects [24-27]. There were also trends toward a lower abundance of Ruminococcus and Coprococcus (Fig. 6), trends also seen in the aforementioned nonhuman primate study [9]. Furthermore, Lachnospira sp. were significantly higher after 2 weeks of an MD (Fig. 2), and evidence has suggested Lachnospira is beneficial in a variety of disease processes, including inflammatory bowel disease and asthma [28, 29].

However, despite the changes that were seen with this intervention, the short-term nature of this analysis (1 month of standardized dietary changes and just 2 weeks of MD) may limit appreciation of the long-term impact of MD as was suggested by Wu et al. [21]. However, this study implies that changes occur quickly to regimented dietary changes. The aforementioned population-based studies represent larger scale data to help guide conclusions; but dietary compliance in any study is challenging, particularly when no food is provided and when dietary trends are self-reported, as is often performed. Therefore, the observed impacts of the controlled dietary intervention performed in this small study warrant further investigation to confirm the rapid change in alpha diversity.

\section{Conclusion}

The variety of positive outcomes seen in the literature with MD warrant further research to elucidate how this diet modifies disease. Data on the effect of fecal microbiota transplant in IBS patients as it relates to microbial alterations have shown mixed results, but targeted and standardized dietary therapy warrants further exploration $[30,31]$. This interventional pilot study utilizing a regimented and controlled dietary intervention provides data to support MD as a mechanism to affect microbial alterations. These data will serve as a basis for further exploration of the impact of an MD on the microbiome as this relates to a variety of disease models, particularly IBS.

\section{Statement of Ethics}

The procedures were approved by the Wake Forest Health Sciences Institutional Review Board (IRB00039422) and registered with ClinicalTrials.gov (NCT03269032). Written informed consent was obtained for each participant.

\section{Conflict of Interest Statement}

There are no conflicts of interest to report.

\section{Funding Sources}

The Department of Internal Medicine/Gastroenterology at Wake Forest University School of Medicine provided funding for this project. We received no payment to write this article. All authors had full access to the full data in the study and accept responsibility to submit for publication.

\section{Author Contributions}

J.R., F.W., R.N., H.Y., and R.W. performed literature search, data analysis, data interpretation, and writing. J.R., F.W., and R.W. performed study design, conceptualization, data curation, investigation methodology, project administration, supervision, and validation.

\section{Data Availability Statement}

Data cannot be shared for confidentiality reasons. Queries about the data should be directed to the corresponding author. 


\section{References}

1 Lynch SV, Pedersen O. The human intestinal microbiome in health and disease. $\mathrm{N}$ Engl J Med. 2016;375(24):2369-79.

2 Gentile CL, Weir TL. The gut microbiota at the intersection of diet and human health. Science. 2018;362(6416):776-80.

3 David LA, Maurice CF, Carmody RN, Gootenberg DB, Button JE, Wolfe BE, et al. Diet rapidly and reproducibly alters the human gut microbiome. Nature. 2014;505(7484):55963.

4 Del Chierico F, Vernocchi P, Dallapiccola B, Putignani L. Mediterranean diet and health food effects on gut microbiota and disease control. Int J Mol Sci. 2014;15(7):11678-99.

5 Garcia-Mantrana I, Selma-Royo M, Alcantara C, Collado MC. Shifts on gut microbiota associated to mediterranean diet adherence and specific dietary intakes on general adult population. Front Microbiol. 2018;9:890.

6 Bailey MA, Holscher HD. Microbiome-mediated effects of the mediterranean diet on inflammation. Adv Nutr. 2018;9(3):193-206.

7 Gutiérrez-Díaz I, Fernández-Navarro T, Sánchez B, Margolles A, González S. Mediterranean diet and faecal microbiota: a transversal study. Food Funct. 2016;7(5):2347-56.

8 De Filippis F, Pellegrini N, Vannini L, Jeffery IB, La Storia A, Laghi L, et al. High-level adherence to a Mediterranean diet beneficially impacts the gut microbiota and associated metabolome. Gut. 2016;65(11):1812-21.

9 Nagpal R, Shively CA, Appt SA, Register TC, Michalson KT, Vitolins MZ, et al. Gut microbiome composition in non-human primates consuming a western or mediterranean diet. Front Nutr. 2018;5:28.

10 Shively CA, Register TC, Appt SE, Clarkson TB, Uberseder B, Clear KYJ, et al. Consumption of mediterranean versus western diet leads to distinct mammary gland microbiome populations. Cell Rep. 2018;25(1):47-57.e3.

11 Tap J, Derrien M, Törnblom H, Brazeilles R Cools-Portier S, Doré J, et al. Identification of an intestinal microbiota signature associated with severity of irritable bowel syndrome. Gastroenterology. 2017;152(1):111-128.e8.
12 Labus JS, Hollister EB, Jacobs J, Kirbach K, Oezguen N, Gupta A, et al. Differences in gut microbial composition correlate with regional brain volumes in irritable bowel syndrome. Microbiome. 2017;5(1):49.

13 Jeffery IB, O'Toole PW, Öhman L, Claesson MJ, Deane J, Quigley EM, et al. An irritable bowel syndrome subtype defined by speciesspecific alterations in faecal microbiota. Gut. 2012;61(7):997-1006.

14 Zito FP, Polese B, Vozzella L, Gala A, Genovese D, Verlezza V, et al. Good adherence to mediterranean diet can prevent gastrointestinal symptoms: a survey from Southern Italy. World J Gastrointest Pharmacol Ther. 2016; 7(4):564-71.

15 Nagpal R, Neth BJ, Wang S, Craft S, Yadav H. Modified mediterranean-ketogenic diet modulates gut microbiome and short-chain fatty acids in association with Alzheimer's disease markers in subjects with mild cognitive impairment. EBioMedicine. 2019;47:529-42.

16 Ahmadi S, Wang S, Nagpal R, Wang B, Jain S, Razazan A, et al. A human-origin probiotic cocktail ameliorates aging-related leaky gut and inflammation via modulating the microbiota/taurine/tight junction axis. JCI Insight. 2020;5(9):e132055.

17 Caporaso JG, Lauber CL, Walters WA, BergLyons D, Huntley J, Fierer N, et al. Ultra-highthroughput microbial community analysis on the Illumina HiSeq and MiSeq platforms. ISME J. 2012;6(8):1621-4.

18 Ahmadi S, Nagpal R, Wang S, Gagliano J, Kitzman DW, Soleimanian-Zad S, et al. Prebiotics from acorn and sago prevent high-fatdiet-induced insulin resistance via microbiome-gut-brain axis modulation. J Nutr Biochem. 2019;67:1-13

19 Caporaso JG, Kuczynski J, Stombaugh J, Bittinger K, Bushman FD, Costello EK, et al. QIIME allows analysis of high-throughput community sequencing data. Nat Methods. 2010; 7(5):335-6

20 Segata N, Izard J, Waldron L, Gevers D, Miropolsky L, Garrett WS, et al. Metagenomic biomarker discovery and explanation. Genome Biol. 2011;12(6):R60.

21 Wu GD, Chen J, Hoffmann C, Bittinger K, Chen YY, Keilbaugh SA, et al. Linking Longterm dietary patterns with gut microbial enterotypes. Science. 2011;334(6052):105-8.
22 Ghosh TS, Rampelli S, Jeffery IB, Santoro A, Neto M, Capri M, et al. Mediterranean diet intervention alters the gut microbiome in older people reducing frailty and improving health status: the NU-AGE 1-year dietary intervention across five European countries. Gut. 2020;69(7):1218-28.

23 Ley RE, Turnbaugh PJ, Klein S, Gordon JI. Microbial ecology: human gut microbes associated with obesity. Nature. 2006;444(7122): 1022-3.

24 Cani PD, De Vos WM. Next-generation beneficial microbes: the case of Akkermansia muciniphila. Front Microbiol. 2017;8:1765.

25 Naito Y, Uchiyama K, Takagi T. A next-generation beneficial microbe: Akkermansia muciniphila. J Clin Biochem Nutr. 2018;63(1): 33-5.

26 Tilg H, Moschen AR. Microbiota and diabetes: an evolving relationship. Gut. 2014;63(9): 1513-21.

27 Shin NR, Lee JC, Lee HY, Kim MS, Whon TW, Lee MS, et al. An increase in the Akkermansiaspp. population induced by metformin treatment improves glucose homeostasis in diet-induced obese mice. Gut. 2014;63(5): 727-35.

28 Wang Y, Gao X, Ghozlane A, Hu H, Li X, Xiao $\mathrm{Y}$, et al. Characteristics of faecal microbiota in paediatric Crohn's disease and their dynamic changes during infliximab therapy. J Crohns Colitis. 2018;12(3):337-46.

29 Stiemsma LT, Arrieta MC, Dimitriu PA, Cheng J, Thorson L, Lefebvre DL, et al. Shifts in Lachnospira and Clostridium sp. in the 3-month stool microbiome are associated with preschool age asthma. Clinical Sci. 2016; 130(23):2199-207.

30 Halkjær SI, Christensen AH, Lo BZS, Browne PD, Günther S, Hansen LH, et al. Faecal microbiota transplantation alters gut microbiota in patients with irritable bowel syndrome: results from a randomised, double-blind placebo-controlled study. Gut. 2018;67(12):210715.

31 El-Salhy M, Hatlebakk JG, Gilja OH, Bråthen Kristoffersen A, Hausken T. Efficacy of faecal microbiota transplantation for patients with irritable bowel syndrome in a randomised, double-blind, placebo-controlled study. Gut. 2020;69(5):859-67. 